\title{
Massless infinite spin (super)particles and fields
}

\author{
I.L. Buchbinder ${ }^{1}$, S. Fedoruk ${ }^{2}$, A.P. Isaev ${ }^{2,3,4}$ \\ ${ }^{1}$ Department of Theoretical Physics, Tomsk State Pedagogical University, \\ 634041 Tomsk, Russia, \\ joseph@tspu.edu.ru \\ ${ }^{2}$ Bogoliubov Laboratory of Theoretical Physics, Joint Institute for Nuclear Research, \\ 141980 Dubna, Moscow Region, Russia, \\ fedoruk@theor.jinr.ru, isaevap@theor.jinr.ru \\ ${ }^{3}$ Faculty of Physics, Lomonosov Moscow State University, 119991 Moscow, Russia \\ ${ }^{4}$ St.Petersburg Department of Steklov Mathematical Institute of RAS, \\ Fontanka 27, 191023 St. Petersburg, Russia
}

\begin{abstract}
A new twistorial field formulation of a massless infinite spin particle is derived. We find a twistorial infinite spin field and derive its helicity decomposition. The twistorial equations of motion for infinite spin fields in the cases of integer and half-integer helicities are derived. We show that the infinite integer-spin field and infinite half-integer-spin field form the $\mathcal{N}=1$ infinite spin supermultiplet. The corresponding supersymmetry transformations are presented. We prove that the supersymmetry algebra is closed on-shell.
\end{abstract}

PACS: 11.10.Ef, 11.30.Cp, 11.30.Pb, 03.65.Pm

Keywords: twistors, infinite spin particles, canonical quantization, supersymmetry

Contribution to the Volume dedicated to the 80-th Anniversary Jubilee of Andrei A. Slavnov 


\section{Introduction}

Principles of symmetry, formulated in terms of group theory, play a remarkable role in theoretical and mathematical physics. It is sufficient to say that the Standard Model is constructed on the base of gauge principle, which imposes the essential restrictions both on classical Lagrangian and on scattering amplitudes. The fundamental contribution to realization of the gauge principle in quantum field theory was done by A.A. Slavnov [1]. The paper under consideration is devoted to some aspects of symmetry related to Poincaré group.

Relativistic symmetry associates the elementary particles with irreducible representations of the Poincaré group $I S O^{\uparrow}(1,3)$ (or its covering $I S L(2, \mathbb{C})$ ). Classification of the $I S O^{\uparrow}(1,3)$ unitary irreducible representations was given in [2, 3, 4]. Unitary irreducible representations of the Poincaré group, which are usually interesting from the physical point of view, act in the space of states with non-negative mass squared $\mathrm{m}^{2} \geq 0$ and non-negative energy $E=k_{0} \geq 0$ (here $k_{0}$ is zero component of 4-momentum of particle).

To characterize these irreducible representations we need to consider the corresponding irreducible representations of the Lie algebra $i s o(1,3)$ with generators $\hat{P}_{n}, \hat{M}^{m k}$ (components of momentum and angular momentum) and defining relations

$$
\begin{aligned}
& {\left[\hat{P}_{n}, \hat{P}_{m}\right]=0, \quad\left[\hat{P}_{n}, \hat{M}_{m k}\right]=i\left(\eta_{k n} \hat{P}_{m}-\eta_{m n} \hat{P}_{k}\right),} \\
& {\left[\hat{M}_{n m}, \hat{M}_{k \ell}\right]=i\left(\eta_{n k} \hat{M}_{m \ell}-\eta_{m k} \hat{M}_{n \ell}+\eta_{m \ell} \hat{M}_{n k}-\eta_{n \ell} \hat{M}_{m k}\right),}
\end{aligned}
$$

where metric tensor is $\left\|\eta_{m k}\right\|=\operatorname{diag}(+1,-1,-1,-1)$.

There are $\mathbf{2}$ classes of physically interesting unitary irreducible representations (irreps) of Poincaré group: 1. massive irreps and 2. massless irreps.

1. Massive irreps. The algebra $i s o(1,3)$ has two Casimir operators $\hat{P}^{n} \hat{P}_{n}$ and $\hat{W}^{n} \hat{W}_{n}$, where

$$
\hat{W}_{n}=\frac{1}{2} \varepsilon_{n m k r} \hat{M}^{m k} \hat{P}^{r}
$$

are components of the Pauli-Lubanski vector which satisfy

$$
\hat{W}_{n} \hat{P}^{n}=0, \quad\left[\hat{W}_{k}, \hat{P}_{n}\right]=0, \quad\left[\hat{W}_{m}, \hat{W}_{n}\right]=i \varepsilon_{m n k r} \hat{W}^{k} \hat{P}^{r} .
$$

On the space of states of massive irreducible representations the Casimir operators are proportional to the unite operator $\mathbb{I}$ :

$$
\hat{P}^{n} \hat{P}_{n}=\mathrm{m}^{2} \mathbb{I} \quad\left(\mathrm{m}^{2}>0\right), \quad \hat{W}^{n} \hat{W}_{n}=-\mathrm{m}^{2} j(j+1) \mathbb{I},
$$

where the real number $\mathrm{m}>0$ is called mass and the real number $j \in \mathbb{Z}_{\geq 0} / 2$ is called spin.

2. Massless irreps. The Casimir operators of $i s o(1,3)$ are

$$
\hat{P}^{n} \hat{P}_{n}=\mathrm{m}^{2}=0, \quad \hat{W}^{2}=\hat{W}^{n} \hat{W}_{n}=-\mu^{2} .
$$

In this case we have two subcases: A) $\mu^{2}=0$ and B) $\mu^{2} \neq 0$.

In massless case A) we obtain usual massless helicity representations with

$$
\hat{W}^{2}=0, \quad \hat{P}^{2}=0, \quad \hat{P}_{n} \hat{W}^{n}=0 \quad \stackrel{\text { for } \mathbb{R}^{1,3}}{\Longrightarrow} \quad \hat{W}_{n}=\hat{\Lambda} \cdot \hat{P}_{n},
$$

where central element $\hat{\Lambda} \in i s o(1,3)$ is called helicity operator and its eigenvalues are $\Lambda=0, \pm 1 / 2, \pm 1, \pm 3 / 2, \ldots$ 
In massless case B) we have

$$
\hat{W}^{2}=-\mu^{2}, \quad \hat{P}^{2}=0, \quad \hat{P}_{n} \hat{W}^{n}=0,
$$

which correspond to massless irreducible representation of infinite (continuous) spin. To describe these representations we have to introduce "canonically conjugate" to $\hat{P}_{k}$, $\hat{W}_{n}$ variables $x_{k}, y_{n}$ :

$$
x=\left(x_{0}, x_{1}, x_{2}, x_{3}\right) \in \mathbb{R}^{1,3}, \quad y=\left(y_{0}, y_{1}, y_{2}, y_{3}\right) \in \mathbb{R}^{1,3} .
$$

Then, as it was shown in [2, 3, 4], the massless infinite spin irreducible representations of the Poincaré group are realized in the space of wave functions (WF) $\Phi(x, y)$ which satisfy the conditions

$$
\begin{array}{ll}
\frac{\partial}{\partial x^{m}} \frac{\partial}{\partial x_{m}} \Phi=0, & \frac{\partial}{\partial x^{m}} \frac{\partial}{\partial y_{m}} \Phi=0, \\
\frac{\partial}{\partial y^{m}} \frac{\partial}{\partial y_{m}} \Phi=\mu^{2} \Phi, & -i y^{m} \frac{\partial}{\partial x^{m}} \Phi=\Phi .
\end{array}
$$

This paper is devoted to some aspects of theory of massless infinite (or continues) spin unitary irreducible representations of the $\operatorname{ISL}(2, \mathbb{C})$ group. Various problems related to the quantummechanical and field descriptions of such states were considered in a wide range of works devoted to particles and fields of infinite (continues) spin (see, e.g., [5]-27]). Motivation of the investigations of the infinite spin representations is caused by an identical spectrum of states of the infinite spin theory [5] and the higher-spin theory [28, 29, 30] (see also the reviews [31, 32, 33]) and by its potential relation to the string theory (see [34] and recent paper [35] and references in it) as candidates for Quantum Gravity Theory.

In our recent papers [36, 37] we constructed a new model of an infinite (continuous) spin particle, which is a generalization of the twistor formulation of standard (with fixed helicity) massless particle [38, 39, 40] to massless infinite spin representations. As a result of a quantization procedure, we obtained infinite spin fields that demonstrate a transparent decomposition of continues spin irreducible representations into infinite sum of states with all helicities. We stress that for massless case $\mathbf{B}$, irreducible representations are not characterized by definite helicities. Besides, using the field twistor transform, we can now get the space-time-spinorial description of infinite spin fields with integer or half-integer helicities that form a supermultiplet of infinite spins [6, 18].

This paper is based on the results obtained in [36, 37].

\section{Wigner-Bargmann space-time formulation}

The Wigner-Bargmann space-time formulation [2, 3, 4] of the irreducible infinite spin massless representation can be realized by means of quantization of the particle model with the following Lagrangian

$$
\mathcal{L}_{\text {sp.-time }}=p_{m} \dot{x}^{m}+q_{m} \dot{y}^{m}+e p_{m} p^{m}+e_{1} p_{m} q^{m}+e_{2}\left(q_{m} q^{m}+\mu^{2}\right)+e_{3}\left(p_{m} y^{m}-1\right),
$$

where $\left\{p_{n}(\tau), q_{n}(\tau)\right\}$ are momenta canonically conjugated to coordinates $\left\{x_{n}(\tau), y_{n}(\tau)\right\}, \tau$ is the evolution parameter and $\dot{x}_{k}(\tau):=\partial_{\tau} x_{k}(\tau)$. The Lagrangian (2.1) yields the canonical Poisson brackets

$$
\left\{x^{m}, p_{n}\right\}=\delta_{n}^{m}, \quad\left\{y^{m}, q_{n}\right\}=\delta_{n}^{m}
$$


and first-class constraints

$$
\begin{gathered}
T:=p_{m} p^{m} \approx 0, \quad T_{1}:=p_{m} q^{m} \approx 0, \\
T_{2}:=q_{m} q^{m}+\mu^{2} \approx 0, \quad T_{3}:=p_{m} y^{m}-1 \approx 0,
\end{gathered}
$$

which correspond to the Wigner-Bargmann equations (1.2). The variables $e(\tau), e_{1}(\tau), e_{2}(\tau), e_{3}(\tau)$ are Lagrange multipliers for the constraints (2.2). Nonvanishing Poisson brackets of the constraints (2.2) are

$$
\left\{T_{1}, T_{3}\right\}=-T, \quad\left\{T_{2}, T_{3}\right\}=-2 T_{1}
$$

The action $S_{s p .-t i m e}=\int d \tau \mathcal{L}_{s p .-t i m e}$ is invariant under the transformations which generated by the quantities

$$
P_{m}=p_{m}, \quad M_{m n}=x_{m} p_{n}-x_{n} p_{m}+y_{m} q_{n}-y_{n} q_{m} .
$$

These charges form the Poincaré algebra with respect to Poisson brackets. We see that additional coordinates $y^{m}$ in the arguments of these fields play the role of spin variables.

Now by making use of constraints $T \approx 0, T_{1} \approx 0, T_{2} \approx 0, T_{3} \approx 0$ we obtain relations

$$
P_{m} P^{m} \approx 0, \quad W_{m} W^{m}=\frac{1}{2} M_{n k} M^{n k} P_{m} P^{m}-M_{m k} M^{n l} P^{k} P_{l} \approx-\mu^{2} .
$$

where $W_{m}=\frac{1}{2} \varepsilon_{m n k l} P^{n} M^{k l}$ are the components of the Pauli-Lubanski pseudovector. Therefore, the model with Lagrangian $\mathcal{L}_{s p \text {-time }}$ indeed describes the massless particle with continuous spin. We note that vectors $q_{m}$ and $W_{m}=\varepsilon_{m n k l} p^{n} y^{l} q^{l}$ do not coincide to each other and components $W_{m}$ strictly speaking are not canonically conjugated to $y_{m}$.

After canonical quantization the constraints (2.2) yield the Wigner-Bargmann equations (1.2) for the continuous spin fields $\Phi(x, y)$.

\section{Twistorial formulation of continuous spin particles.}

Our aim is to reformulate the Wigner-Bargmann model with Lagrangian (2.1) in terms of twistor variables. More precisely we need to construct a twistor particle model which is classically equivalent to the Wigner-Bargmann model with Lagrangian $\mathcal{L}_{\text {sp.-time }}$.

Below we will use the following two-spinor conventions about notation. The totally antisymmetric tensor $\epsilon^{m n k l}$ has the component $\epsilon^{0123}=1$. We use the set of $\sigma$-matrices: $\sigma^{n}=\left(\sigma^{0} \equiv\right.$ $\left.I_{2}, \sigma^{1}, \sigma^{2}, \sigma^{3}\right)$ and the set of dual $\sigma$-matrices: $\tilde{\sigma}^{n}=\left(\sigma^{0},-\sigma^{1},-\sigma^{2},-\sigma^{3}\right)$, where $\sigma^{i}$ are usual Pauli matrices. We also use standard van der Waerden spinor notation with dotted and undotted spinor indices and raise and lower them by means of metrics: $\epsilon_{\alpha \beta}, \epsilon_{\dot{\alpha} \dot{\beta}}$ and their inverse $\epsilon^{\alpha \beta}$, $\epsilon^{\dot{\alpha} \dot{\beta}}$ with components $\epsilon_{12}=-\epsilon_{21}=1$. In particular $\left(\tilde{\sigma}_{m}\right)^{\dot{\alpha} \beta}=\epsilon^{\dot{\alpha} \dot{\delta}} \epsilon^{\beta \gamma}\left(\sigma_{m}\right)_{\gamma \dot{\delta}}$. The links between the Minkowski four-vectors and spinorial quantities are

$$
A_{\alpha \dot{\beta}}=\frac{1}{\sqrt{2}} A_{m}\left(\sigma^{m}\right)_{\alpha \dot{\beta}}, \quad A^{\dot{\alpha} \beta}=\frac{1}{\sqrt{2}} A_{m}\left(\tilde{\sigma}^{m}\right)^{\dot{\alpha} \beta}, A_{m}=\frac{1}{\sqrt{2}} A_{\alpha \dot{\beta}}\left(\tilde{\sigma}_{m}\right)^{\dot{\beta} \alpha},
$$

so that $A^{m} B_{m}=A_{\alpha \dot{\beta}} B^{\dot{\beta} \alpha}$.

In [36, 37] we propose that the twistorial formulation of the infinite (continuous) spin particle is described by the bosonic Weyl spinors

$$
\pi_{\alpha}, \quad \bar{\pi}_{\dot{\alpha}}:=\left(\pi_{\alpha}\right)^{*}, \quad \rho_{\alpha}, \quad \bar{\rho}_{\dot{\alpha}}:=\left(\rho_{\alpha}\right)^{*},
$$


and their canonically conjugated spinors

$$
\omega^{\alpha}, \quad \bar{\omega}^{\dot{\alpha}}:=\left(\omega^{\alpha}\right)^{*}, \quad \eta^{\alpha}, \quad \bar{\eta}^{\dot{\alpha}}:=\left(\eta^{\alpha}\right)^{*} .
$$

The nonzero Poisson brackets of these spinors are

$$
\left\{\omega^{\alpha}, \pi_{\beta}\right\}=\left\{\eta^{\alpha}, \rho_{\beta}\right\}=\delta_{\beta}^{\alpha}, \quad\left\{\bar{\omega}^{\dot{\alpha}}, \bar{\pi}_{\dot{\beta}}\right\}=\left\{\bar{\eta}^{\dot{\alpha}}, \bar{\rho}_{\dot{\beta}}\right\}=\delta_{\dot{\beta}}^{\dot{\alpha}} .
$$

Twistorial Lagrangian of the infinite (continuous) spin particle is written in the form [36, 37]:

$$
\mathcal{L}_{\text {twistor }}=\pi_{\alpha} \dot{\omega}^{\alpha}+\bar{\pi}_{\dot{\alpha}} \dot{\bar{\omega}}^{\dot{\alpha}}+\rho_{\alpha} \dot{\eta}^{\alpha}+\bar{\rho}_{\dot{\alpha}} \dot{\bar{\eta}}^{\dot{\alpha}}+l \mathcal{M}+k \mathcal{U}+\ell \mathcal{F}+\bar{\ell} \overline{\mathcal{F}},
$$

where $l(\tau), k(\tau), \ell(\tau), \bar{\ell}(\tau)$ are the Lagrange multipliers for the constraints

$$
\begin{aligned}
\mathcal{M} & :=\pi^{\alpha} \rho_{\alpha} \bar{\rho}_{\dot{\alpha}} \bar{\pi}^{\dot{\alpha}}-\mu^{2} / 2 \approx 0, \\
\mathcal{F} & :=\eta^{\alpha} \pi_{\alpha}-1 \approx 0, \quad \overline{\mathcal{F}}:=\bar{\pi}_{\dot{\alpha}} \bar{\eta}^{\dot{\alpha}}-1 \approx 0, \\
\mathcal{U} & :=i\left(\omega^{\alpha} \pi_{\alpha}-\bar{\pi}_{\dot{\alpha}} \bar{\omega}^{\dot{\alpha}}+\eta^{\alpha} \rho_{\alpha}-\bar{\rho}_{\dot{\alpha}} \bar{\eta}^{\dot{\alpha}}\right) \approx 0,
\end{aligned}
$$

One can check that the first-class constraints (3.4), (3.4), (3.4) generate abelian Lie group which acts in the phase space of spinors (3.1), (3.2) as follows:

$$
\begin{gathered}
\left(\begin{array}{ll}
\pi_{1} & \rho_{1} \\
\pi_{2} & \rho_{2}
\end{array}\right) \rightarrow\left(\begin{array}{cc}
\pi_{1} & \rho_{1} \\
\pi_{2} & \rho_{2}
\end{array}\right)\left(\begin{array}{cc}
e^{i \beta} & \alpha e^{i \beta} \\
0 & e^{i \beta}
\end{array}\right) \\
\left(\begin{array}{ll}
\eta_{1} & \omega_{1} \\
\eta_{2} & \omega_{2}
\end{array}\right) \rightarrow\left(\begin{array}{ll}
\eta_{1} & \omega_{1} \\
\eta_{2} & \omega_{2}
\end{array}\right)\left(\begin{array}{cc}
e^{-i \beta} & -\alpha e^{-i \beta} \\
0 & e^{-i \beta}
\end{array}\right)+\frac{2}{\mu^{2}}\left(\bar{\rho}_{\dot{\alpha}} \bar{\pi}^{\dot{\alpha}}\right)\left(\begin{array}{cc}
\pi_{1} & \rho_{1} \\
\pi_{2} & \rho_{2}
\end{array}\right)\left(\begin{array}{cc}
\gamma & 0 \\
0 & -\gamma
\end{array}\right)
\end{gathered}
$$

and the transformations that are complex conjugation of (3.7), (3.8), where $\beta(\tau), \gamma(\tau) \in \mathbb{R}$ and $\alpha(\tau) \in \mathbb{C} \backslash 0$ are the parameters of the gauge group generated by constraints (3.4), (3.4), (3.4).

Proposition 1. The Wigner-Bargmann space-time (2.1) and twistorial (3.3) formulations of the infinite (continuous) spin particle are equivalent on the classical level by means of the generalized Cartan-Penrose relations [38, 39, 40]

$$
p_{\alpha \dot{\beta}}=\pi_{\alpha} \bar{\pi}_{\dot{\beta}}, \quad q_{\alpha \dot{\beta}}=\pi_{\alpha} \bar{\rho}_{\dot{\beta}}+\rho_{\alpha} \bar{\pi}_{\dot{\beta}}
$$

and by the following generalized incidence relations [38, 39, 40]:

$$
\begin{array}{cl}
\omega^{\alpha}=\bar{\pi}_{\dot{\alpha}} x^{\dot{\alpha} \alpha}+\bar{\rho}_{\dot{\alpha}} y^{\dot{\alpha} \alpha}, & \bar{\omega}^{\dot{\alpha}}=x^{\dot{\alpha} \alpha} \pi_{\alpha}+y^{\dot{\alpha} \alpha} \rho_{\alpha}, \\
\eta^{\alpha}=\bar{\pi}_{\dot{\alpha}} y^{\dot{\alpha} \alpha}, & \bar{\eta}^{\dot{\alpha}}=y^{\dot{\alpha} \alpha} \pi_{\alpha} .
\end{array}
$$

The proof of this Proposition is straightforward and is given in [36, 37].

\section{Quantization of the twistorial model and twistor field of the infinite spin particle}

Quantization of the model is drastically simplified if we introduce new spinorial variables by means of Bogolyubov canonical transformations (cf. gauge transformations (3.7), (3.8)):

$$
\left(\begin{array}{ll}
\pi_{1} & \rho_{1} \\
\pi_{2} & \rho_{2}
\end{array}\right)=\sqrt{M}\left(\begin{array}{cc}
p_{1}^{(z)} & 0 \\
p_{2}^{(z)} & p^{(s)} / p_{1}^{(z)}
\end{array}\right)\left(\begin{array}{cc}
1 & p^{(t)} \\
0 & 1
\end{array}\right)
$$




$$
\left(\begin{array}{ll}
\eta_{1} & \omega_{1} \\
\eta_{2} & \omega_{2}
\end{array}\right)=\left(\begin{array}{cc}
0 & z_{1} / \sqrt{M} \\
-t / \pi_{1} & z_{2} / \sqrt{M}
\end{array}\right)\left(\begin{array}{cc}
1 & -p^{(t)} \\
0 & 1
\end{array}\right)+\frac{s}{M}\left(\begin{array}{ll}
\pi_{1} & \rho_{1} \\
\pi_{2} & \rho_{2}
\end{array}\right)\left(\begin{array}{cc}
1 & 0 \\
0 & -1
\end{array}\right)
$$

where $M=\mu / \sqrt{2}$ and new variables are defined by the expressions

$$
\begin{gathered}
p_{\alpha}^{(z)}=\pi_{\alpha} / \sqrt{M}, \quad p^{(s)}=\pi^{\alpha} \rho_{\alpha} / M, \quad p^{(t)}=\rho_{1} / \pi_{1} \\
\omega^{\alpha}=\frac{1}{\sqrt{M}} z^{\alpha}-\frac{1}{M} s \rho^{\alpha}-\frac{\delta^{\alpha 1}}{\pi_{1}} t p^{(t)} \\
\eta^{\alpha}=\frac{1}{M} s \pi^{\alpha}+\frac{\delta^{\alpha 1}}{\pi_{1}} t
\end{gathered}
$$

By means of complex conjugation we obtain the conjugated coordinates $\bar{z}^{\dot{\alpha}}, \bar{s}, \bar{t}$ and their momenta $\bar{p}_{\dot{\alpha}}^{(z)}, \bar{p}^{(s)}, \bar{p}^{(t)}$. The nonzero canonical Poisson brackets of the new variables are

$$
\left\{z^{\alpha}, p_{\beta}^{(z)}\right\}=\delta_{\beta}^{\alpha}, \quad\left\{\bar{z}^{\dot{\alpha}}, \bar{p}_{\dot{\beta}}^{(z)}\right\}=\delta_{\dot{\beta}}^{\dot{\alpha}}, \quad\left\{s, p^{(s)}\right\}=\left\{\bar{s}, \bar{p}^{(s)}\right\}=1, \quad\left\{t, p^{(t)}\right\}=\left\{\bar{t}, \bar{p}^{(t)}\right\}=1 .
$$

In terms of new variables (4.1) the constraints (3.4), (3.5), (3.6) of spinorial model (3.3) take very simple form

$$
\begin{aligned}
\mathcal{M}^{\prime} & :=p^{(s)} \bar{p}^{(s)}-1 \approx 0 \\
\mathcal{F}^{\prime} & :=t-1 \approx 0, \quad \overline{\mathcal{F}}^{\prime}:=\bar{t}-1 \approx 0 \\
\mathcal{U}^{\prime} & :=\frac{i}{2}\left(z^{\alpha} p_{\alpha}^{(z)}-\bar{z}^{\dot{\alpha}} \bar{p}_{\dot{\alpha}}^{(z)}\right)+i\left(s p^{(s)}-\bar{s} \bar{p}^{(s)}\right) \approx 0 .
\end{aligned}
$$

After canonical quantization $[.,]=.i\{.,$.$\} these constraints turn into equations of motion$

$$
\begin{aligned}
& \left(p^{(s)} \bar{p}^{(s)}-1\right) \Psi^{(c)}=0 \\
& \frac{\partial}{\partial p^{(t)}} \Psi^{(c)}=\frac{\partial}{\partial \bar{p}^{(t)}} \Psi^{(c)}=-i \Psi^{(c)} \\
& {\left[\frac{1}{2}\left(p_{\alpha}^{(z)} \frac{\partial}{\partial p_{\alpha}^{(z)}}-\bar{p}_{\dot{\alpha}}^{(z)} \frac{\partial}{\partial \bar{p}_{\dot{\alpha}}^{(z)}}\right)+p^{(s)} \frac{\partial}{\partial p^{(s)}}-\bar{p}^{(s)} \frac{\partial}{\partial \bar{p}^{(s)}}\right] \Psi^{(c)}=c \Psi^{(c)},}
\end{aligned}
$$

where differential operators in their left hand sides are quantum counterparts of the constraints (4.2), (4.3), (4.4). In equations (4.5), (4.6), (4.7) wave function (or spinorial field)

$$
\Psi^{(c)}\left(p_{\alpha}^{(z)}, \bar{p}_{\dot{\alpha}}^{(z)} ; p^{(s)}, \bar{p}^{(s)} ; p^{(t)}, \bar{p}^{(t)}\right)
$$

is taken in "momentum representation" and describes physical states, which form the space of irreducible representation of Poincaré group with continues spin. The constant $c$ is related to the ambiguity of operator ordering in equation (4.7). In other words, constant $c$ is an analog of the vacuum energy in the quantum oscillator model.

Equations of motion (4.5), (4.6) can be solved explicitly in the form

$$
\Psi^{(c)}=\delta\left(p^{(s)} \cdot \bar{p}^{(s)}-1\right) e^{-i\left(p^{(t)}+\bar{p}^{(t)}\right)} \sum_{k=-\infty}^{\infty} e^{-i k \varphi} \tilde{\psi}^{(c+k)}\left(p^{(z)}, \bar{p}^{(z)}\right)
$$


where $e^{i \varphi}:=\left(p^{(s)} / \bar{p}^{(s)}\right)^{1 / 2}$. Due to the constraint (4.7) the coefficient functions $\tilde{\psi}^{(c+k)}\left(p_{z}, \bar{p}_{z}\right)$ satisfy the equations

$$
\frac{1}{2}\left(p_{\alpha}^{(z)} \frac{\partial}{\partial p_{\alpha}^{(z)}}-\bar{p}_{\dot{\alpha}}^{(z)} \frac{\partial}{\partial \bar{p}_{\dot{\alpha}}^{(z)}}\right) \tilde{\psi}^{(c+k)}=(c+k) \tilde{\psi}^{(c+k)}
$$

Now we can restore the dependence of the wave function (4.8) on the twistor variables. As result we obtain the following statement.

Proposition 2. The twistor wave function which is general solution of the equations of motion (4.5), 4.6), (4.7) is represented in the form

$$
\Psi^{(c)}(\pi, \bar{\pi} ; \rho, \bar{\rho})=\delta\left((\pi \rho)(\bar{\rho} \bar{\pi})-M^{2}\right) e^{-i\left(\frac{\rho_{1}}{\pi_{1}}+\frac{\bar{\rho}_{1}}{\bar{\pi}_{1}}\right)} \hat{\Psi}^{(c)}(\pi, \bar{\pi} ; \rho, \bar{\rho}),
$$

where we make use the shorthand notation $(\pi \rho):=\pi^{\beta} \rho_{\beta},(\bar{\rho} \bar{\pi}):=\bar{\rho}_{\dot{\beta}} \bar{\pi}^{\dot{\beta}}$ and

$$
\hat{\Psi}^{(c)}(\pi, \bar{\pi} ; \rho, \bar{\rho})=\psi^{(c)}(\pi, \bar{\pi})+\sum_{k=1}^{\infty}(\bar{\rho} \bar{\pi})^{k} \psi^{(c+k)}+\sum_{k=1}^{\infty}(\pi \rho)^{k} \psi^{(c-k)}
$$

The coefficient functions $\psi^{(c \pm k)}(\pi, \bar{\pi})$ obey the condition

$$
\Lambda \cdot \psi^{(c \pm k)}(\pi, \bar{\pi})=-(c \pm k) \psi^{(c \pm k)}(\pi, \bar{\pi}),
$$

where $\Lambda=-\frac{1}{2}\left(\pi_{\alpha} \frac{\partial}{\partial \pi_{\alpha}}-\bar{\pi}_{\dot{\alpha}} \frac{\partial}{\partial \bar{\pi}_{\dot{\alpha}}}\right)$ is the helicity operator.

In view of condition (4.12), to describe the bosonic infinite spin representation related to all integer helicities, we put

$$
c=0
$$

and therefore consider the twistorial field $\Psi^{(0)}(\pi, \bar{\pi} ; \rho, \bar{\rho})$. Note that complex conjugate field $\bar{\Psi}^{(0)}$ also has zero charge $c=0$. Similarly, to describe the infinite spin representation related to half-integer helicities we take for $c$ the value

$$
c=-\frac{1}{2}
$$

In view of condition (4.12), the corresponding wave function $\Psi^{(-1 / 2)}(\pi, \bar{\pi} ; \rho, \bar{\rho})$ contains in its expansion only half-integer helicities. The complex conjugate field $\bar{\Psi}^{(+1 / 2)}(\pi, \bar{\pi} ; \rho, \bar{\rho})$ possesses the charge $c=+1 / 2$.

Proposition 3. The twistor wave function $\Psi^{(c)}(\pi, \bar{\pi} ; \rho, \bar{\rho})$, defined in Proposition 2, describes the massless particle of the infinite (continuous) spin:

$$
W^{\alpha \dot{\gamma}} W_{\alpha \dot{\gamma}} \cdot \Psi^{(c)}=-\mu^{2} \Psi^{(c)}
$$

where $W_{\alpha \dot{\gamma}}=\frac{1}{\sqrt{2}} W_{m}\left(\sigma^{m}\right)_{\alpha \dot{\gamma}}$ is the Pauli-Lubański operator

$$
W_{\alpha \dot{\gamma}}=\pi_{\alpha} \bar{\pi}_{\dot{\gamma}} \Lambda+\frac{1}{2}\left[\pi_{\alpha} \bar{\rho}_{\dot{\gamma}}\left(\bar{\pi}_{\dot{\beta}} \frac{\partial}{\partial \bar{\rho}_{\dot{\beta}}}\right)-\rho_{\alpha} \bar{\pi}_{\dot{\gamma}}\left(\pi_{\beta} \frac{\partial}{\partial \rho_{\beta}}\right)\right]+\frac{1}{2}\left[(\bar{\rho} \bar{\pi}) \pi_{\alpha} \frac{\partial}{\partial \bar{\rho}^{\dot{\gamma}}}-(\pi \rho) \bar{\pi}_{\dot{\gamma}} \frac{\partial}{\partial \rho^{\alpha}}\right] \text {. }
$$


Proof. Substitute (4.14) into (4.13) and make use representation (4.10)and equations of motion (4.5), (4.6), (4.7) written it terms of twistor variables

$$
\begin{gathered}
i \pi_{\alpha} \frac{\partial}{\partial \rho_{\alpha}} \Psi^{(c)}=\Psi^{(c)}, \quad i \bar{\pi}_{\dot{\alpha}} \frac{\partial}{\partial \bar{\rho}_{\dot{\alpha}}} \Psi^{(c)}=\Psi^{(c)} \\
\left(\pi_{\alpha} \frac{\partial}{\partial \pi_{\alpha}}-\bar{\pi}_{\dot{\alpha}} \frac{\partial}{\partial \bar{\pi}_{\dot{\alpha}}}+\rho_{\alpha} \frac{\partial}{\partial \rho_{\alpha}}-\bar{\rho}_{\dot{\alpha}} \frac{\partial}{\partial \bar{\rho}_{\dot{\alpha}}}\right) \Psi^{(c)}=2 c \Psi^{(c)} .
\end{gathered}
$$

Recall that the twistorial wave function $\Psi^{(c)}$ is complex and therefore all component fields $\psi^{(c \pm k)}(\pi, \bar{\pi})$ in its expansion are also complex. In view of this we must consider together with the field $\Psi^{(c)}$ its complex conjugated field $\left(\Psi^{(c)}\right)^{*}:=\bar{\Psi}^{(-c)}$ which has the opposite charge $c \rightarrow-c$.

\section{Twistor transform for infinite spin fields}

In this section, we establish a correspondence between twistor fields and fields defined in the four-dimensional Minkowski space-time.

For further convenience we introduce the dimensionless spinor

$$
\xi_{\alpha}:=M^{-1 / 2} \rho_{\alpha}, \quad \bar{\xi}_{\dot{\alpha}}:=M^{-1 / 2} \bar{\rho}_{\dot{\alpha}} .
$$

Then, the twistor wave function $\Psi^{(c)}$ of infinite integer-spin particle (4.10) for $c=0$ can be represented in the form [37]

$$
\begin{aligned}
\Psi^{(0)}(\pi, \bar{\pi} ; \xi, \bar{\xi})= & \delta((\pi \xi)(\bar{\xi} \bar{\pi})-M) e^{-i q_{0} / p_{0} \hat{\Psi}^{(0)}(\pi, \bar{\pi} ; \xi, \bar{\xi})} \\
& \hat{\Psi}^{(0)}=\psi^{(0)}(\pi, \bar{\pi})+\sum_{k=1}^{\infty}(\bar{\xi} \bar{\pi})^{k} \psi^{(k)}(\pi, \bar{\pi})+\sum_{k=1}^{\infty}(\pi \xi)^{k} \psi^{(-k)}(\pi, \bar{\pi}) .
\end{aligned}
$$

In the expansion of $\hat{\Psi}^{(0)}$, all components $\psi^{(k)}(\pi, \bar{\pi})(k \in \mathbb{Z})$ in general are complex functions (fields). Moreover, the quantity $p_{0} / q_{0}$ is expressed by means of the generalized Cartan-Penrose representations (3.9) in spinorial form as

$$
\frac{q_{0}}{p_{0}}=\frac{\sqrt{M} \sum_{\alpha=\dot{\alpha}}\left(\pi_{\alpha} \bar{\xi}_{\dot{\alpha}}+\xi_{\alpha} \bar{\pi}_{\dot{\alpha}}\right)}{\sum_{\beta=\dot{\beta}} \pi_{\beta} \bar{\pi}_{\dot{\beta}}} .
$$

In the case $c=-1 / 2$, the wave function of the infinite half-integer spin particle is

$$
\begin{aligned}
& \Psi^{\left(-\frac{1}{2}\right)}(\pi, \bar{\pi} ; \xi, \bar{\xi})= \delta((\pi \xi)(\bar{\xi} \bar{\pi})-M) e^{-i q_{0} / p_{0} \hat{\Psi}^{\left(-\frac{1}{2}\right)}(\pi, \bar{\pi} ; \xi, \bar{\xi})} \\
& \hat{\Psi}^{\left(-\frac{1}{2}\right)}=\psi^{\left(-\frac{1}{2}\right)}(\pi, \bar{\pi})+\sum_{k=1}^{\infty}(\bar{\xi} \bar{\pi})^{k} \psi^{\left(-\frac{1}{2}+k\right)}(\pi, \bar{\pi})+\sum_{k=1}^{\infty}(\pi \xi)^{k} \psi^{\left(-\frac{1}{2}-k\right)}(\pi, \bar{\pi})
\end{aligned}
$$

The expansion of the complex conjugated wave function $\bar{\Psi}^{\left(+\frac{1}{2}\right)}$ has the form

$$
\begin{aligned}
& \bar{\Psi}^{\left(+\frac{1}{2}\right)}(\pi, \bar{\pi} ; \xi, \bar{\xi})= \delta((\pi \xi)(\bar{\xi} \bar{\pi})-M) e^{i q_{0} / p_{0} \hat{\bar{\Psi}}^{\left(+\frac{1}{2}\right)}(\pi, \bar{\pi} ; \xi, \bar{\xi})} \\
& \hat{\bar{\Psi}}^{\left(+\frac{1}{2}\right)}=\bar{\psi}^{\left(\frac{1}{2}\right)}(\pi, \bar{\pi})+\sum_{k=1}^{\infty}(\bar{\xi} \bar{\pi})^{k} \bar{\psi}^{\left(\frac{1}{2}+k\right)}(\pi, \bar{\pi})+\sum_{k=1}^{\infty}(\pi \xi)^{k} \bar{\psi}^{\left(\frac{1}{2}-k\right)}(\pi, \bar{\pi})
\end{aligned}
$$


where the component fields $\bar{\psi}^{(r)}(\pi, \bar{\pi})$ are complex conjugation of the component fields $\psi^{(-r)}(\pi, \bar{\pi})$ :

$$
\left(\psi^{\left(-\frac{1}{2}+k\right)}\right)^{*}=\bar{\psi}^{\left(\frac{1}{2}-k\right)}, \quad k \in \mathbb{Z} .
$$

\subsection{The case of integer spins}

In this case the $\mathrm{U}(1)$-charge is zero, $c=0$, and the space-time wave function is determined by means of the integral Fourier transformation of twistor field $\Psi^{(0)}(\pi, \bar{\pi} ; \xi, \bar{\xi})$ :

$$
\Phi(x ; \xi, \bar{\xi})=\int d^{4} \pi e^{i p_{\alpha \dot{\alpha}} x^{\dot{\alpha} \alpha}} \Psi^{(0)}(\pi, \bar{\pi} ; \xi, \bar{\xi})=\int d^{4} \pi e^{i \pi_{\alpha} \bar{\pi}_{\dot{\alpha}} x^{\dot{\alpha} \alpha}} \Psi^{(0)}(\pi, \bar{\pi} ; \xi, \bar{\xi}),
$$

where we have used the representation $p_{\alpha \dot{\alpha}}=\pi_{\alpha} \bar{\pi}_{\dot{\alpha}}$ and perform integration over the measure $d^{4} \pi:=\frac{1}{2} d \pi_{1} \wedge d \pi_{2} \wedge d \bar{\pi}_{\dot{1}} \wedge d \bar{\pi}_{\dot{2}}=d \phi d^{4} p \delta\left(p^{2}\right)$ (here $\phi$ is common phase in $\pi_{\alpha}$ which is not presented in $p_{\alpha \dot{\alpha}}=\pi_{\alpha} \bar{\pi}_{\dot{\alpha}}$ ).

Proposition 4. The field $\Phi(x ; \xi, \bar{\xi})$ defined by the integral transformation (5.4) in coordinate representation satisfies four equations

$$
\begin{gathered}
\partial^{\alpha \dot{\alpha}} \partial_{\alpha \dot{\alpha}} \Phi(x ; \xi, \bar{\xi})=0, \quad\left(i \frac{\partial}{\partial \xi_{\alpha}} \partial_{\alpha \dot{\alpha}} \frac{\partial}{\partial \bar{\xi}_{\dot{\alpha}}}-M\right) \Phi(x ; \xi, \bar{\xi})=0, \\
\left(i \xi^{\alpha} \partial_{\alpha \dot{\alpha}} \bar{\xi}^{\dot{\alpha}}+M\right) \Phi(x ; \xi, \bar{\xi})=0, \quad\left(\xi_{\alpha} \frac{\partial}{\partial \xi_{\alpha}}-\bar{\xi}_{\dot{\alpha}} \frac{\partial}{\partial \bar{\xi}_{\dot{\alpha}}}\right) \Phi(x ; \xi, \bar{\xi})=0 .
\end{gathered}
$$

Proof. Make use the integral transformation (5.4) and equations of motion (4.15), (4.16) for $c=0$.

\subsection{The case of half-integer spins}

In this case the $\mathrm{U}(1)$-charge equals $c=-1 / 2$. Then we use the standard prescription of the twistorial definition of space-time fields with nonvanishing helicities. Namely, we have to insert the twistorial spinor $\pi_{\alpha}$ in the integrand in the Fourier transformation:

$$
\Phi_{\alpha}(x ; \xi, \bar{\xi})=\int d^{4} \pi e^{i p_{\beta \dot{\beta}} x^{\dot{\beta} \beta}} \pi_{\alpha} \Psi^{(-1 / 2)}(\pi, \bar{\pi} ; \xi, \bar{\xi})=\int d^{4} \pi e^{i \pi_{\beta} \bar{\pi}_{\dot{\beta}} x^{\dot{\beta} \beta}} \pi_{\alpha} \Psi^{(-1 / 2)}(\pi, \bar{\pi} ; \xi, \bar{\xi}),
$$

and obtain the external spinor index $\alpha$. Then the complex conjugate twistorial field with charge $c=+1 / 2$ is defined analogously

$$
\bar{\Phi}_{\dot{\alpha}}(x ; \xi, \bar{\xi})=\int d^{4} \pi e^{-i \pi_{\beta} \bar{\pi}_{\dot{\beta}} x^{\dot{\beta} \beta}} \bar{\pi}_{\dot{\alpha}} \bar{\Psi}^{(+1 / 2)}(\pi, \bar{\pi} ; \xi, \bar{\xi})
$$

Proposition 5. The space-time fields $\Phi_{\alpha}(x ; \xi, \bar{\xi})$ and $\bar{\Phi}_{\dot{\alpha}}(x ; \xi, \bar{\xi})$, which correspond to the states with half-integer helicities, satisfy massless Dirac-Weyl equations

$$
\partial^{\dot{\alpha} \alpha} \Phi_{\alpha}(x ; \xi, \bar{\xi})=0, \quad \partial^{\dot{\alpha} \alpha} \bar{\Phi}_{\dot{\alpha}}(x ; \xi, \bar{\xi})=0
$$


and integer spin equations:

$$
\begin{aligned}
& \left(i \xi^{\beta} \partial_{\beta \dot{\beta}} \bar{\xi}^{\dot{\beta}}+M\right) \Phi_{\alpha}(x ; \xi, \bar{\xi})=0, \quad\left(i \xi^{\beta} \partial_{\beta \dot{\beta}} \bar{\xi}^{\dot{\beta}}-M\right) \bar{\Phi}_{\dot{\alpha}}(x ; \xi, \bar{\xi})=0, \\
& \left(i \frac{\partial}{\partial \xi_{\beta}} \partial_{\beta \dot{\beta}} \frac{\partial}{\partial \bar{\xi}_{\dot{\beta}}}-M\right) \Phi_{\alpha}(x ; \xi, \bar{\xi})=0, \quad\left(i \frac{\partial}{\partial \xi_{\beta}} \partial_{\beta \dot{\beta}} \frac{\partial}{\partial \bar{\xi}_{\dot{\beta}}}+M\right) \bar{\Phi}_{\dot{\alpha}}(x ; \xi, \bar{\xi})=0, \\
& \left(\xi_{\beta} \frac{\partial}{\partial \xi_{\beta}}-\bar{\xi}_{\dot{\beta}} \frac{\partial}{\partial \bar{\xi}_{\dot{\beta}}}\right) \Phi_{\alpha}(x ; \xi, \bar{\xi})=0, \quad\left(\xi_{\beta} \frac{\partial}{\partial \xi_{\beta}}-\bar{\xi}_{\dot{\beta}} \frac{\partial}{\partial \bar{\xi}_{\dot{\beta}}}\right) \bar{\Phi}_{\dot{\alpha}}(x ; \xi, \bar{\xi})=0 .
\end{aligned}
$$

Proof. Make use the integral transformations (5.6), (5.7) and equations of motion (4.15), (4.16) for $c= \pm 1 / 2$.

We stress that although the twistorial fields $\Psi^{(-1 / 2)}(\pi, \bar{\pi} ; \xi, \bar{\xi})$ and $\bar{\Psi}^{(+1 / 2)}(\pi, \bar{\pi} ; \xi, \bar{\xi})$ have nonvanishing charges $c=\mp 1 / 2$, their integral transformed versions $\Phi_{\dot{\alpha}}(x ; \xi, \bar{\xi})$ and $\bar{\Phi}_{\dot{\alpha}}(x ; \xi, \bar{\xi})$ have zero $\mathrm{U}(1)$-charge. This fact is crucial for forming infinite spin supermultiplets, as we will see below.

\section{$6 \quad$ Infinite spin supermultiplet}

We unify fields $\Phi(x ; \xi, \bar{\xi})$ and $\Phi_{\alpha}(x ; \xi, \bar{\xi})$ with integer and half-integer helicities into one supermultiplet. The fields $\Phi(x ; \xi, \bar{\xi})$ and $\Phi_{\alpha}(x ; \xi, \bar{\xi})$ contain the bosonic $\psi^{(k)}(\pi, \bar{\pi})$ and fermionic $\psi^{(k-1 / 2)}(\pi, \bar{\pi})$ component fields $(k \in \mathbb{Z})$ with all integer and half-integer spins, respectively.

It is natural to expect that individual components $\psi^{(k)}(\pi, \bar{\pi})$ and $\psi^{(k-1 / 2)}(\pi, \bar{\pi})$ of these fields should form the on-shell $\mathcal{N}=1$ higher spin supermultiplet. Therefore, the bosonic (even) $\Phi(x ; \xi, \bar{\xi})$ and fermionic (odd) $\Phi_{\alpha}(x ; \xi, \bar{\xi})$ fields themselves should form the on-shell $\mathcal{N}=1$ infinite spin supermultiplet containing an infinite number of conventional supermultiplets.

Similar to the Wess-Zumino supermultiplet (see, e.g., [41, 42]), we write supersymmetry transformations of the fields $\Phi$ and $\Phi_{\alpha}$ in the form

$$
\delta \Phi=\varepsilon^{\alpha} \Phi_{\alpha}, \quad \delta \Phi_{\alpha}=2 i \bar{\varepsilon}^{\dot{\beta}} \partial_{\alpha \dot{\beta}} \Phi
$$

where $\varepsilon_{\alpha}, \bar{\varepsilon}_{\dot{\alpha}}$ are the constant odd Weyl spinors. The commutators of these transformations are

$$
\begin{aligned}
& \left(\delta_{1} \delta_{2}-\delta_{2} \delta_{1}\right) \Phi=-2 i a^{\beta \dot{\beta}} \partial_{\beta \dot{\beta}} \Phi \\
& \left(\delta_{1} \delta_{2}-\delta_{2} \delta_{1}\right) \Phi_{\alpha}=-2 i a^{\beta \dot{\beta}} \partial_{\beta \dot{\beta}} \Phi_{\alpha}+2 i a_{\alpha \dot{\beta}} \partial^{\dot{\beta} \beta} \Phi_{\beta},
\end{aligned}
$$

where $a_{\alpha \dot{\beta}}:=\varepsilon_{1 \alpha} \bar{\varepsilon}_{2 \dot{\beta}}-\varepsilon_{2 \alpha} \bar{\varepsilon}_{1 \dot{\beta}}$. As we see, the superalgebra (6.2) is closed on-shell on the generator

$$
P_{\beta \dot{\beta}}=-i \partial_{\beta \dot{\beta}}
$$

due to the Dirac-Weyl equations of motion (5.8). Moreover, the whole system of equations of motion (5.5), (5.8), (5.9) is invariant with respect to supersymmetry transformations (6.2).

Using the inverse integral Fourier transformations, we rewrite (6.2) as supersymmetry transformations for the twistor fields $\Psi^{(0)}(\pi, \bar{\pi} ; \xi, \bar{\xi}), \Psi^{(-1 / 2)}(\pi, \bar{\pi} ; \xi, \bar{\xi})$ in the momentum representation:

$$
\delta \Psi^{(0)}=\varepsilon^{\alpha} \pi_{\alpha} \Psi^{(-1 / 2)}, \quad \delta \Psi^{(-1 / 2)}=-2 \bar{\varepsilon}^{\dot{\alpha}} \pi_{\dot{\alpha}} \Psi^{(0)}
$$


For the bosonic $\psi^{(k)}(\pi, \bar{\pi})$ and fermionic $\psi^{\left(-\frac{1}{2}+k\right)}(\pi, \bar{\pi})$ twistorial components at all $k \in \mathbb{Z}$ we have

$$
\delta \psi^{(k)}=\varepsilon^{\alpha} \pi_{\alpha} \psi^{\left(-\frac{1}{2}+k\right)}, \quad \delta \psi^{\left(-\frac{1}{2}+k\right)}=-2 \bar{\varepsilon}^{\dot{\alpha}} \pi_{\dot{\alpha}} \psi^{(k)} .
$$

The bosonic field $\psi^{(k)}$ and fermionic field $\psi^{\left(-\frac{1}{2}+k\right)}$ at fixed $k \in \mathbb{Z}$ describe massless states with helicities $(-k)$ and $\left(\frac{1}{2}-k\right)$, respectively. Thus, the infinite-component supermultiplet of the infinite spin stratifies into an infinite number of levels with pairs of the fields $\psi^{(k)}, \psi^{\left(-\frac{1}{2}+k\right)}$ at fixed $k \in \mathbb{Z}$. The supersymmetry transforms the bosonic and fermionic fields into each other inside a given level $k$. The boosts of the Poincare group transform the levels with different $k$ and therefore mix the fields with different values of $k$.

In final we point out that superfield description of infinite spin supermultiplet was presented in recent paper [43].

\section{$7 \quad$ Summary and outlook}

Let us summarize the obtained results.

- We have presented the new twistorial formulation of the massless infinite spin particles and fields.

- We gave the helicity decomposition of twistorial infinite spin fields and constructed the field twistor transform to define the space-time infinite (continuous) spin fields $\Phi(x ; \xi, \bar{\xi})$ and $\Phi_{\alpha}(x ; \xi, \bar{\xi})$.

- We found the equations of motion for $\Phi(x ; \xi, \bar{\xi})$ and $\Phi_{\alpha}(x ; \xi, \bar{\xi})$ and showed that these fields form the $\mathcal{N}=1$ infinite spin supermultiplet.

- A natural question arises about status of such fields in Lagrangian field theory and also about possibility to construct self-consistent interaction of such fields. One of the commonly used methods for this purpose is the BRST approach, which was used in the case of continuous spin particles in [12], [21] - [25]. In a recent paper [23] the covariant Lagrangian formulation of the infinite integer-spin field was constructed by using the methods developed in [44, 45].

\section{Acknowledgments}

S.F. and A.P.I. thank M. Podoinitsyn for helpful discussion of results of this paper. I.L.B. and A.P.I. acknowledge the partial support of the Ministry of Science and High Education of Russian Federation, project No.3.1386.2017. I.L.B. acknowledges the support of the Russian Foundation for Basic Research, project No.18-02-00153. A.P.I. acknowledges the support of the Russian Science Foundation, grant No. 19-11-00131. 


\section{References}

[1] A.A. Slavnov, Ward identities in gauge theories, Theor. Math. Phys. 10, 2 (1972) 99 (Teor. Mat. Fiz. 10, 2 (1972) 153); Invariant regularization of gauge theories, Theor. Math. Phys., 13, 2 (1972) 1064 (Teor. Mat. Fiz. 13, 2 (1972) 174).

[2] E.P. Wigner, On unitary representations of the inhomogeneous Lorentz group, Annals Math. 40 (1939) 149.

[3] E.P. Wigner, Relativistische Wellengleichungen, Z. Physik 124 (1947) 665.

[4] V.Bargmann, E.P. Wigner, Group theoretical discussion of relativistic wave equations, Proc. Nat. Acad. Sci. US 34 (1948) 211.

[5] G.J. Iverson, G. Mack, Quantum fields and interactions of massless particles - the continuous spin case, Annals Phys. 64 (1971) 253.

[6] L. Brink, A.M. Khan, P. Ramond, X.-Z. Xiong, Continuous spin representations of the Poincaré and superPoincaré groups, J. Math. Phys. 43 (2002) 6279, arXiv: hep-th/0205145.

[7] X. Bekaert, N. Boulanger, The unitary representations of the Poincaré group in any spacetime dimension, Lectures presented at 2nd Modave Summer School in Theoretical Physics, 6-12 Aug 2006, Modave, Belgium, arXiv:hep-th/0611263.

[8] X. Bekaert, J. Mourad, The continuous spin limit of higher spin field equations, JHEP 0601 (2006) 115, arXiv:hep-th/0509092.

[9] P. Schuster, N. Toro, On the theory of continuous-spin particles: wavefunctions and soft-factor scattering amplitudes, JHEP 1309 (2013) 104, arXiv:1302.1198 [hep-th].

[10] P.Schuster, N. Toro, On the theory of continuous-spin particles: helicity correspondence in radiation and forces, JHEP 1309 (2013) 105, arXiv:1302.1577 [hep-th] .

[11] P. Schuster, N. Toro, A gauge field theory of continuous-spin particles, JHEP 1310 (2013) 061, arXiv:1302.3225 [hep-th].

[12] A.K.H. Bengtsson, BRST Theory for Continuous Spin, JHEP 1310 (2013) 108, arXiv:1303.3799 [hep-th].

[13] P. Schuster, N. Toro, A CSP field theory with helicity correspondence, Phys. Rev. D91 (2015) 025023, arXiv:1404.0675 [hep-th].

[14] V.O. Rivelles, Gauge theory formulations for continuous and higher spin fields, Phys. Rev. D91 (2015) 125035, arXiv:1408.3576 [hep-th].

[15] X. Bekaert, M. Najafizadeh, M.R.Setare, A gauge field theory of fermionic Continuous-Spin Particles, Phys. Lett. B760 (2016) 320, arXiv:1506.00973 [hep-th].

[16] R.R. Metsaev, Continuous spin gauge field in (A)dS space, Phys. Lett. B767 (2017) 458, arXiv:1610.00657[hep-th]. 
[17] R.R. Metsaev, Fermionic continuous spin gauge field in (A)dS space, Phys. Lett. B773 (2017) 135, arXiv:1703.05780 [hep-th].

[18] Yu.M.Zinoviev, Infinite spin fields in $d=3$ and beyond, Universe 3 (2017) 63, arXiv:1707.08832 [hep-th].

[19] X. Bekaert, E.D.Skvortsov, Elementary particles with continuous spin, Int. J. Mod. Phys. A32 (2017) 1730019, arXiv:1708.01030 [hep-th].

[20] M.V. Khabarov, Yu.M.Zinoviev, Infinite (continuous) spin fields in the frame-like formalism, Nucl. Phys. B928 (2018) 182, arXiv:1711.08223 [hep-th].

[21] K.B. Alkalaev, M.A. Grigoriev, Continuous spin fields of mixed-symmetry type, JHEP 1803 (2018) 030, arXiv:1712.02317 [hep-th].

[22] R.R. Metsaev, BRST-BV approach to continuous-spin field, Phys. Lett. B781 (2018) 568, arXiv:1803.08421 [hep-th] .

[23] I.L. Buchbinder, V.A. Krykhtin, H. Takata, BRST approach to Lagrangian construction for bosonic continuous spin field, Phys. Lett. B785 (2018) 315, arXiv:1806.01640 [hep-th] .

[24] K. Alkalaev, A. Chekmenev, M. Grigoriev, Unified formulation for helicity and continuous spin fermionic fields, JHEP 1811 (2018) 050, arXiv:1808.09385 [hep-th].

[25] R.R. Metsaev, Cubic interaction vertices for massive/massless continuous-spin fields and arbitrary spin fields, JHEP 1812 (2018) 055, arXiv:1809.09075 [hep-th].

[26] V.O.Rivelles, A gauge field theory for continuous spin tachyons, arXiv:1807.01812 [hep-th] .

[27] R.R. Metsaev, Light-cone continuous-spin field in AdS space, Phys. Lett. B 793 (2019) 134; arXiv:1903.10495 [hep-th].

[28] M.A. Vasiliev, Consistent equations for interacting massless fields of all spins in the first order in curvatures, Annals Phys. 190 (1989) 59.

[29] M.A. Vasiliev, Algebraic aspects of the higher spin problem, Phys. Lett. B257 (1991) 111.

[30] M.A. Vasiliev, More on equations of motion for interacting massless fields of all spins in (3+1)-dimensions, Phys. Lett. B285 (1992) 225.

[31] M.A. Vasiliev, Progress in higher spin gauge theories, Proceedings of the International Conference on Quantization, Gauge Theory, and Strings: Conference Dedicated to the Memory of Prof. E. Fradkin, Eds. A. Semikhatov, M. Vasiliev, V. Zaikin, Scientific World, Moscow, 2001, 452-472, arXiv: hep-th/0104246.

[32] M.A. Vasiliev, Relativity, causality, locality, quantization and duality in the Sp $(2 M)$ invariant generalized spacetime, in Multiple Facets of Quantization and Supersymmetry, Michael Marinov Memorial Volume, Eds. M. Olshanetsky and A. Vainshtein, World Scientific, 2002, 826-872, arXiv:hep-th/0111119. 
[33] X. Bekaert, S. Cnockaert, C. Iazeolla, M.A. Vasiliev, Nonlinear higher spin theories in various dimensions, Proceedings of the 1st Solvay Workshop on Higher Spin Gauge Theories, 12 14 May 2004. Brussels, Belgium, Eds. R. Argurio, G. Barnich, G. Bonelli, M. Grigoriev, Int. Solvay Institutes, 2006, 132-197, arXiv:hep-th/0503128.

[34] J. Mund, B.Schroer, J. Yngvason, String localized quantum fields from Wigner representations, Phys. Lett. B596 (2004) 156, arXiv:math-ph/0402043.

[35] M.A.Vasiliev, From Coxeter higher-spin theories to strings and tensor models, arXiv:1804.06520 [hep-th].

[36] I.L. Buchbinder, S. Fedoruk, A.P. Isaev, A.Rusnak, Model of massless relativistic particle with continuous spin and its twistorial description, JHEP 1807 (2018) 031, arXiv:1805.09706 [hep-th].

[37] I.L. Buchbinder, S. Fedoruk, A.P. Isaev, Twistorial and space-time descriptions of massless infinite spin (super)particles and fields, Nucl. Phys. B 945 (2019) 114660, arXive: 1903.07947 [hep-th].

[38] R. Penrose, Twistor algebra, J. Math. Phys. 8 (1967) 345.

[39] R. Penrose, M.A.H. MacCallum, Twistor theory: an approach to the quantization of fields and spacetime, Phys. Rept. 6 (1972) 241.

[40] R.Penrose, W.Rindler, Spinors And Space-time. Vol. 2: Spinor And Twistor Methods In Space-time Geometry, Cambridge University Press, 1988, 512 pages.

[41] J. Wess, J. Bagger, Supersymmetry and supergravity, Princeton, NJ, USA: Princeton University Press, 1992, 259 pages.

[42] I.L. Buchbider, S.M. Kuzenko, Ideas and Methods of Supersymmetry and Supergravity, IOP Publ., 1998, 656 pages.

[43] I.L. Buchbinder, S.J. Gates, K. Koutrolikos, Superfield continuous spin equations of motion, Phys. Lett. B793 (2019) 445, arXiv:1903.08631 [hep-th].

[44] I.L. Buchbinder, V.A. Krykhtin, A. Pashnev, BRST approach to Lagrangian construction for fermionic massless higher spin fields, Nucl. Phys. B711 (2005) 367, arXiv: hep-th/0410215.

[45] I.L. Buchbinder, V.A. Krykhtin, Gauge invariant Lagrangian construction for massive bosonic higher spin fields in D dimensions, Nucl. Phys. B727 (2005) 537, arXiv:hep-th/0505092. 